\title{
A note on multiple schedules of IRT reinforcement
}

Hichard W. Malott 2 and William W. Cumming COLUMBIA UNIVERSITY

\begin{abstract}
Data were obtained with rats on the effects of interresponse time (IRT) contingent reinforcement of the lever press response using schedules in which two intervals of reinforced IRTs were associated with two different stimulus light intensities $\left(\mathrm{S}^{+}\right.$values) and reinforcement was withheld in the presence of an intermediate intensity $\left(\mathrm{S}^{-}\right)$. When the reinforcement intervals were the same for the two $\mathrm{S}^{+}$values, the response rates were essentially the same for all three stimulus intensities; however when the reinforcement intervals were different, the response rates in the presence of $\mathrm{S}^{-}$were sometimes intermediate and sometimes lowest. The height of the mode of the IRT distributions was consistently lowest for the distributions obtained in $\mathrm{S}^{-}$.
\end{abstract}

\section{Problem}

Zimmerman \& Schuster (1962) have demonstrated that when different schedules of IRT reinforcement are correlated with different values of an extroceptive stimulus, the IRT distributions normally associated with those schedules also become correlated with the extroceptive stimuli. Using similar schedules of reinforcement, Hernstein (1962) showed that $\mathrm{S}^{-}$stimulus values intermediate between $\mathrm{S}^{+}$values generate response rates which are intermediate between the rates associated with the $\mathrm{S}^{+}$values. The present research is a further investigation of multiple schedules of IRT reinforcement.

\section{Method}

Twelve male albino rats were used. They were fed at the end of each session and were maintained at approximately $80 \%$ of their extrapolated normal weight. They had previously served in a multiple $\mathrm{S}^{-}$experimentusing the same schedule of reinforcement as in the present experiment.

A Grason-Stadler test chamber was modified to contain a liquid dipper, the cup of which was normally within the chamber. The dipper was used to deliver a drop of Eagle Brand sweetened condensed milk which served as the reinforcement for the lever press response. In addition, a false ceiling consisting of three sheets of white diffusing plastic were placed beneath 25 7 watt lamps. By varying the number of lamps which were turned on, the overall illumination of the test chamber could be controlled. The programing and recording circuitry were housed in an adjoining room.

The sessions were $50 \mathrm{~min}$. in length. The Ss had previously beem divided into groups of two and assigned different pairs of reinforcement intervals. The lower bounds of these intervals are shown above the associated light intensity $\left(\mathrm{S}^{+}\right)$along the top of each set of coordinates in Fig. 1. The intervals were unbounded on the upper end, so that any IRT falling above the lower bound was reinforced. Three stimulus intensities (shown along the abscissa of Fig. 1) were presented in a mixed order at the rate of one every minute. For all groups, the middle intensity served as $\mathrm{S}^{-}$.

The data presented in the next section are the average of two sessions and follow seven sessions exposure to the reinforcement and stimulus presentation schedules just described. Missing data are due either to death of the S or apparatus failure.

\section{Results and Diseussion}

The response rates were essentially constant over the three stimulus intensities for the groups with the same lower bounds in both $\mathrm{S}^{+}$values; this was expected on the basis of Hernstein's (1962) findings, although he used more than two $\mathrm{S}^{+}$values and never had the same schedule of reinforcement associated with different $\mathrm{S}^{+}$values. For the 16 and $4 \mathrm{sec}$. lower bounds group, the rate at the intermediate intensity $\left(\mathrm{S}^{-}\right)$was intermediate between that obtained at the two $\mathrm{S}^{+}$intensities; this is again in keeping with Hernstein's results. Contradicting this, however, is the finding that the rate in $\mathrm{S}^{-}$was minimal for the 8 and $2 \mathrm{sec}$. group.

For the groups with different lower bound values in the $\mathrm{S}^{+}$stimuli, the mode of the IRT distribution was sometimes in the vicinity of the shorter lower bound even when the longer lower bound was in effect. The mode was

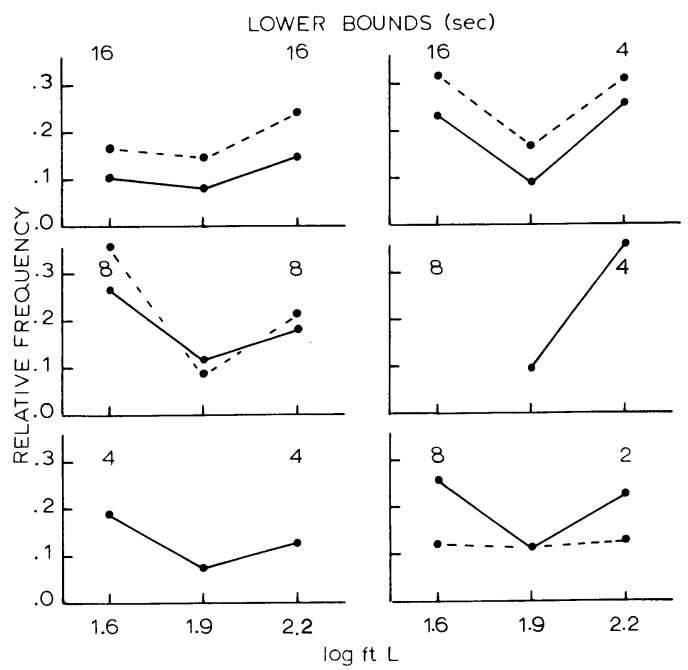

Fig. 1. The height of the mode of the IRT distributions as a function of the lower bound of reinforced IRT interval and the intensity of test chamber illumination. The distributions were computed with IRT class intervals equal in width to $1 / 8$ of the lower bound nearest the mode. 
generally in that vicinity during the $\mathrm{S}^{-}$stimulus also. The height of this mode associated with the various schedules and stimulus intensities is shown in Fig. 1. While there is no consistent difference between the two $\mathrm{S}^{+}$values, the mode in $\mathrm{S}^{-}$is always the lowest. This pattern is consistent in spite of the differences in the rates described above.

\section{Reply}

Dr. Longstreth's comments ask two basic questions: (1) Were the ratio scores justified? Is it possible that pre-test differences may have contaminated the ratio measures and thereby pioduced the differences which we attribute to the reinforcement effect of the tone? (2) How do we justify our conclusion in view of the fact that the no-shock, no-tone control group was higher than any experimental group?

The first question can be answered by an examination of the pre-test data. The number of responses made by the different groups during the half-hour pre-test run does show some variability. That was the reason for our use of ratios. Figure 1 illustrates the raw data. The deviant groups are the 2-shock-tone experimental group and the 20-shock control group. If the pre-test rates alone affected the post/pre-test ratios in the final data analysis, the 2-shock-tone group should be higher than the other experimental group; however, this group is not significantly different from the other groups. The 20shock control group should be lower than its experimental group; they a re not significantly different. In fact, in both cases the direction of the differences is in the

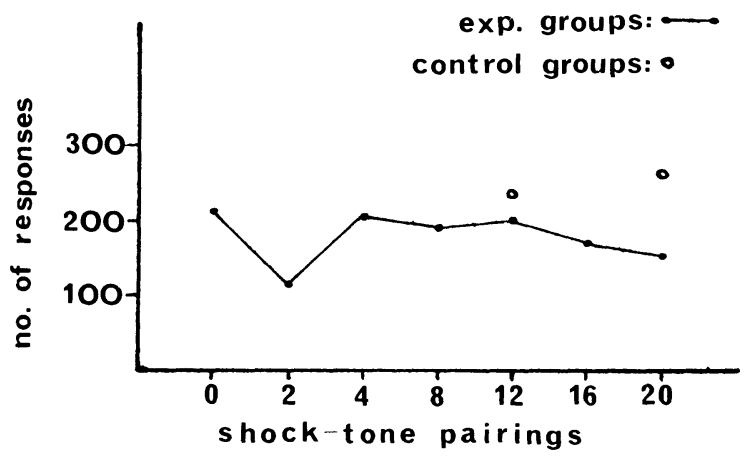

Fig. 1. The mean number of responses made during the pre-shock 30-min. period of free responding.
Referenees

HERNSTEIN, R. J. Method for sensory scaling with animals. Science, $1962,135,40-41$.

ZIMMERMAN, J., \& SCHUSTER, C. R. Spaced responding in multiple DRL schedules. J. exp. Anal. Behav., 1962, 5, 497-504. Notes

1. This research was supported by Public Health Service Grant MH-06911-01.

2. Now at Denison University, Granville, Ohio.

opposite direction. In the light of this data, we feel that the use of ratio scores is perfectly justified.

Dr. Longstreth also questions our statistical analysis and suggests that we need an "... overall test of difference in means..." We certainly would not have considered using the $t$ and Tukey A test before performing an analysis of variance. The $F$ was 4.57 with 6 and 28 degrees of freedom. Such an $F$ justifies all of our tests between selected pairs of means. The Tukey A test was used because it lowers the possibility of a Type 1 error when making several intergroup comparisons. Despite the conservative nature of the test, our differences were significant.

The differences between our experimental and 0shock-tone control groups are basic to the whole idea of our study. Since shock exposure, per se, lowers the post-shock rates, we feel that the reinforcing effects of a post-shock stimulus can only be determined by comparing animals who have been exposed to shock. In this case, we are comparing Ss who have all been exposed to a treatment, which severely affects the response rate. Thus the difference between our 12-shock-tone and 12-shock-0 groups shows that the post-shock tone raised the already depressed rate. If we compared the 12-shock-tone group to a no-shock group, the reinforcing effects of the tone would be masked because of the depression produced by the shock.

If, as Dr. Longstreth suggests, the tone itself were facilitory, we would expect the other experimental groups to show more responses to extinction. They were significantly below the 12-shock-tone group, despite the fact that some experimental Ss had more experience with the tone. Thus we believe this criticism is invalid. In short, we do not find any suggestions in these comments that invalidate our conclusions.

William Wagman Joseph D. Allen Southern Illinois University 